\title{
Neurotoxic Snake Envenomation: Neostigmine-Induced Paradoxical Weakness
}

\author{
I. K. Karthika ${ }^{1} \cdot$ Amit Kumar Satapathy ${ }^{1}$
}

Received: 5 August 2020 / Accepted: 2 December 2020 / Published online: 8 January 2021

(C) Dr. K C Chaudhuri Foundation 2021

To the Editor: Children admitted with neurotoxic snake envenomation is a life-threating condition but can have an excellent prognosis if managed early with anti-snake venom and life-support measures. But we came across an unanticipated pitfall while managing a neurotoxic snake envenomation case where the child improved but then he went into neuromuscular paralysis after almost $12 \mathrm{~h}$ of recovery. We want to highlight a significant, avoidable but unreported event in management of neurotoxic snake envenomation - neostigmineinduced paradoxical weakness.

An 8-y-old boy presented with a history of neurotoxic snake envenomation (probably king Cobra) over right forearm. At presentation, he had ptosis, poor respiratory effort, and altered sensorium (Glasgow Coma Scale of 7/15) for which he required mechanical ventilation. He was managed as per snake-bite management protocol proposed by WHO. Anti-snake venom (ASV) was started soon after admission along with neostigmine and atropine and other supportive care. He had received total 25 vials of Indian polyvalent ASV. Atropine was given at a dose of $0.05 \mathrm{mg} / \mathrm{kg}$ followed by neostigmine $0.04 \mathrm{mg} / \mathrm{kg}$ IV initially, followed by $0.03 \mathrm{mg} / \mathrm{kg}$ in subsequent doses as per protocol in view of positive response. Child recovered in next $12 \mathrm{~h}$, was successfully weaned off from ventilator support and oxygen as well. After $8 \mathrm{~h}$ of extubation, he developed drowsiness along with shallow breathing pattern. There was no history of any seizure episodes. Blood gas revealed respiratory acidosis with carbon dioxide retention ( $\left.\mathrm{pH} 7.12, \mathrm{PCO}_{2}: 85 \mathrm{~mm}, \mathrm{HCO}_{3}: 28.3\right)$. He was reintubated and started on mechanical ventilation in view of poor respiratory effort. Gradually the child recovered completely after $6 \mathrm{~h}$ of reintubation with no residual neurological deficits.

Amit Kumar Satapathy

amitkumar.satapathy@yahoo.co.in

1 Department of Pediatrics, All India Institute of Medical Sciences, Bhubaneswar, Odisha 751 019, India
The cause of worsening after complete recovery was unexpected in a child with neurotoxic snake envenomation. Though initially we attributed to hypoxic brain injury, seizure or aspiration but subsequent clinical evaluation and investigation did not suggest so (chest radiograph and computer tomography brain normal). On retrospective analysis of case record, it was noticed that worsening was temporally related to administration of neostigmine which was given inadvertently as 6 hourly in first $24 \mathrm{~h}$ even after complete recovery. On literature review, neostigmine is known to cause paradoxical muscle weakness, predominantly involving respiratory muscles when doses are administered after complete recovery of neuromuscular paralysis [1,2]. The adverse physiologic effects of neostigmine after complete neuromuscular recovery usually reported at higher doses but evidence for same at a dose $<0.03 \mathrm{mg} / \mathrm{kg}$ is not clear [3]. Our child received a cumulative dose of $0.22 \mathrm{mg} / \mathrm{kg}$ (total of $4.2 \mathrm{mg}$ ) in $24 \mathrm{~h}$. Hence, we want to highlight the issue of careful administration of neostigmine under strict cardio-respiratory monitoring in children with neurotoxic snake envenomation to prevent catastrophic event, moreover when child is recovering.

\section{Compliance with Ethical Standards}

Conflict of Interest None.

\section{References}

1. Naguib M, Kopman AF. Neostigmine-induced weakness: what are the facts? Anaesthesia. 2018;73(9):1055-7.

2. Phillips S, Stewart PA. Catching a unicorn: neostigmine and muscle weakness-not neostigmine for all, but quantitative monitoring for everyone! Anesthesiology. 2018;129:381-2.

3. Luo J, Chen S, Min S, Peng L. Reevaluation and update on efficacy and safety of neostigmine for reversal of neuromuscular blockade. Ther Clin Risk Manag. 2018;14:2397-406.

Publisher's Note Springer Nature remains neutral with regard to jurisdictional claims in published maps and institutional affiliations. 